\title{
Redesigning the Open-Access Institutional Repository: A User Experience Approach
}

\author{
Edward Luca and Bhuva Narayan \\ University of Technology Sydney, Australia \\ edward.luca@uts.edu.au, bhuva.narayan@uts.edu.au
}

\begin{abstract}
This paper details how a university library evaluated its institutional repository using a user experience design (UXD) methodology and redesigned it based on the findings. The online repository, running on DSpace, was not being utilized as expected by academics and researchers, so a detailed user evaluation and usability study was undertaken to find out the reasons why. Findings showed lack of usability and a mismatch between user expectations and system architecture. Hence, significant improvements were made to the user interface, and in communicating the status of items held in the repository (open or closed access). The authors assess the impact of these changes and argue that better usability results in greater visibility of the open-access repository, and hence, greater visibility for the university's researchers. Other challenges regarding the adoption of open access by academics and researchers at the university are also discussed.
\end{abstract}

Keywords: Open access - Institutional repositories · User experience . Usability · Digital collections · Digital libraries

\section{Introduction}

Institutional repositories (IRs) are a valuable proposition for universities. They showcase a university's research output, increase the visibility of their scholars' research, increase citation counts, and are a measure of an institution's prestige. They are also one of the fastest growing kinds of digital libraries, with many scholars arguing that IRs have significant potential to reshape the scholarly landscape and advance the open-access movement [1]. As a low-cost way of archiving and disseminating content, it would seem that there is a clear opportunity for IRs to break down the barriers of access to scholarly communication. Despite such promise, IRs are often under-utilized and infrequently accessed, and developing buy-in from faculty members is an especially challenging reality $[2]$.

\section{Literature Review}

Academics already have numerous administrative duties that constrain their research and writing time, and the perception of IR deposits as an administrative 
task means that academics do not clearly understand the benefits of OA repositories [3]. Bell, Foster and Gibbons argue that the issue of populating the repository with full-text materials is the most significant barrier to the success of an IR [4]. Librarians are often tasked with providing support to faculties for research data services, though many do not have sufficient training in understanding issues such as data storage, discoverability, and the workflow of researchers [5]. Librarians, often the managers or curators of these repositories, approach scholarship from a very different perspective to researchers. They see research outputs as an organizational resource to be managed [6], and are largely unaware of issues that limit academic participation in the IR, such as tenure, career development, and academic freedom. Repository software packages such as DSpace, Digital Commons, and e-Prints are often time-consuming and complicated to install and configure [7]. Koshiyama et al. conducted usability testing to correct usability and information architecture problems with their institution's repository and found that issues "such as labelling, size of source, buttons nomenclature, lack of system feedback" [11] could be identified by allowing a representative sample of users to identify issues or validate design decisions.

For a digital library to be truly useful, designers must adopt a user-centered perspective and understand who their users are, what they require of the system, and any prior knowledge that influences their behaviour [8]. While much has been written about the users of digital libraries in general, there is little research examining the accessibility and usability of institutional repositories from the end-user's perspective [9]. Aljohani and Blustein write that understanding the needs of repository users would "play a vital role in increasing the acceptability and effectiveness of the IR systems" [10]. They argue that students do not commonly use IRs because the system has not been designed with their needs in mind. To address this lack of understanding, they propose developing personas, or user profiles, to inform decision-making, refine the design of the system, and limit goals around what users actually need [10]. Despite this need for changing perceptions about institutional repositories, the process first involves an understanding of the current environment within each institution.

\section{Context}

The issues described in the literature review above led us to consider an approach utilizing user experience methodologies to redesign the University of Technology Sydney's (UTS) institutional repository. An archived version of the old site is located at http://web.archive.org/web/20140301103148/http:// epress.lib.uts.edu.au/research. It is the second-ranked repository in Australasia (Webometric Oceania Rankings) for the size of its open-access collection, with more than 30 percent of the 35,000 publications stored available as open access. UTS uses a current research information system (CRIS) called Symplectic Elements to manage its research outputs. Researchers can log in to Symplectic Elements and 'claim' their publications if they are the authors. The system supports UTS's Open Access Policy, which mandates that all UTS research outputs 
must be collected and stored in the repository [14]. Nevertheless, the adoption of the system was low and the previous version of the UTS IR was very dated and unappealing to use. There was very little incentive for academics to ensure their work was made available through it, and librarians were cautious to promote a clunky and awkward system. The UTS Library team sought to address many of these issues in its redesign of their institutional repository: including findability, navigability, and overall user experience.

\section{Methodology}

User experience (UX) has become an increasingly valued framework for examining library technologies from the user's perspective. While the definition and scope of UX is still contested, scholars generally agree that UX is dynamic, context-dependent, and subjective [12]. Blandford and Buchanan identify "effectance" as an aspect of the user experience meaning "the user's sense of satisfaction at having achieved an interesting effect" [13]. In the context of digital libraries, a good user experience may not necessarily be about finding the perfect document, but rather about serendipity and information discovery - locating new, interesting or even surprising materials. Focusing on user experience allows us to examine more non-utilitarian qualities of the system, including meaning, affect and value. It can be difficult to translate these emotional and psychological needs into design decisions, but examining the usability, navigability, findability and accessibility of a system can inform the redesign of its features, user interface, and interaction design. This is an area that is yet to be thoroughly explored in the literature on IRs so far [2], and this paper argues that the principles of UX design can be utilized to greatly enhance the experience of using an institutional repository.

As part of this process, UTS Library conducted a user experience analysis using two complementary approaches. The first was a Heuristic Evaluation method as detailed by Jakob Nielsen [19], wherein a small set of evaluators, no more than 6-8 people [21] examine the interface and judge its compliance with recognized usability principles (the "heuristics"), which are: (1) visibility of system status, (2) match between system and the real world, (3) user control and freedom, (4) consistency and standards, and (5) error prevention. The second was the User and the Task-by-Type taxonomy by Ben Shneiderman [20], which specify that a good visualization of a digital library should provide: (1) an overview of the collection, (2) allow users to zoom in on items of interest, (3) allow users to filter out uninteresting items, (4) allow details on demand for selected items, (5) allow users to view relationships among items, (6) allow users to keep a history of actions, and (7) allow users to extract a subcollection through search parameters. 


\section{Results}

Two Information Science researchers, two Information Science students, and two librarians, in accordance with the three stakeholder personas we were targeting, participated in the study. We conducted a paired co-discovery and analysis with dyadic participant pairs who sat down together to evaluate the system: researcher-librarian, researcher-student, librarian-researcher, and librarianstudent. The user experience study included usability analysis, metadata recommendations for SEO discovery, navigability, accessibility and design recommendations. Below is a summary of the findings that relate to the redesign:

- The repository had no identity or branding and looked like a generic database.

- The interface was centered around searching rather than browsing, with a search box or basic listing of research areas as the only navigation option.

- Jargon or terminology used was never obvious or explained, for example, DSpace's Community $>$ Collection structure which did not make sense to many of our researchers.

- The interface was cluttered with too much information, including a lot of metadata and statistics.

- While all of the communities and browsing categories were visible on the homepage, it was not made clear to the user what the context of these items was within the site.

- The organization of the site's content, and the way in which it was presented, gave no clear direction for the user as to where they should begin looking for content. Most users skipped straight to the search box, which impeded discovery.

- There were a number of issues flagged in terms of the handling of and display of meta-tags within the $<$ head $>$ of the website's code, which caused issues with search engines finding our repository's content. While it is known that Google does not rely on this metadata for indexing, it does rely on it for the display of the site's information and important subpages in a user's search results. Further to this, both OJS and DSpace display the Dublin Core metadata of indexed articles within the $<$ head $>$ data of the file, but this does not support the indexing of the article as stated above.

- While Dublin Core (DC) is a good schema to use as a standard recognized by all institutions, Google Scholar ignores DC terms in favor of Highwire Press Tags. Highwire Press Tags are recommended for IRs and can be enabled in both DSpace and OJS.

- According to Google Scholar's Webmaster Guidelines for indexing, it is not just the web pages that need to be optimized for indexing, but also the PDF files themselves. As academic researchers are generally responsible for adding their own research into the database (Symplectic Elements), this posed a series of issues in terms of how to get researchers to comply with recommendations for $\mathrm{PDF}$ optimization prior to uploading them into the system.

All of the issues identified above were subsequently addressed in the redesign of the repository. 


\section{Discussion and Implementation}

The new interface was designed over a period of three months. An interdepartmental collaboration was an essential part of the process, as each department (information services, IT services, eScholarship services, and graphic design), offered its own insights and perspectives into the design process. As part of this process the repository was re-branded as Open Publications of UTS Scholars (OPUS), which is now located at https://opus.lib.uts.edu.au/ and other changes were implemented as below.

\subsection{Visual Design}

The project team decided to implement a 'sunburst' design on the OPUS homepage and at the item level. A sunburst is where items in a hierarchy are laid out radially: the top of the hierarchy sits at the center, and deeper levels are further away. Stasko et al. found that the sunburst method is useful in conveying structure and hierarchy, and well-received by participants in their study [15]. The slices of the sunburst are categorized by the UTS Organizational Groups, which typically follows the structure of Faculty $>$ School. The slices scale dynamically depending on the number of items, which visually communicates the size of the collection. The sunburst is also useful in orienting users who have arrived at a specific article within the OPUS collection. Users can easily 'drill up' from this point to access new materials or see related items.

\subsection{Organizational System}

In the previous version of the UTS eRepository, research was categorized according to Field of Research (FOR) codes, which are an Australian classification of research disciplines [16]. Although this categorization of university research was convenient for university accounting purposes, it was not always understood by the researchers themselves or by users. Hence, a decision was made to instead display the Faculty $>$ School organizational structure of the university in OPUS to assist researchers familiar with UTS.

\subsection{Terminology}

Our user studies confirmed that the terminology we used for each item state were unclear to our users. This is a common issue in the design of library systems, where librarians frequently use jargon such as 'hold request', 'InterLibrary loan', and so on, which mean little to the average user. Thong, Ham and Tam argue that often there is a disconnect between the vocabulary users use to express their information needs, and the terminology of the designers of digital libraries [17]. Using natural language, elaborating or explaining confusing terms, and being consistent with terms all help to encourage correct choices by users, by reducing the cognitive barriers caused by the complexity of library resources [18]. 
To address this issue, we designed a visualization of the 'Copyright Clearance Process' which not only explained the current state of an item, but also offered an indication of the process in granting access to items. By indicating some form of progression or workflow, the IR offers the user much more information about the state of objects, and also lets them know if they need to check back at a later date to access an item. The definitions of each item state were also explained in the footer of the pages. A Help page was introduced with information around how Symplectic feeds into OPUS, with a diagram explaining the workflow process for researchers.

\section{Conclusion and Future Directions}

Through adopting a user experience design approach and conducting a thorough usability study of our eRepository interface, we were able to redesign the DSpace interface and customize it for our institution. The resulting web interface is more intuitive and meaningful for our academics and our researchers, and also aids information discovery online for others looking for papers on any given topic through internet searches. The long-term impact of the redesign is hard to assess at this early stage, but there is some Google Analytics data to suggest that users spend more time on OPUS and download more items than in the previous version. We are unlikely to draw any substantial conclusions without a full year's worth of data. For the future, there are a number of functions on DSpace that are not implemented in OPUS which we would like to explore. These include the ability to export citations, and for digital for theses, the ability to link to the supervisor's profile. We would also like to provide further statistics to the user, including download rates for publications. We plan to conduct a qualitative study using interviews to understand other factors that limit engagement by academics. Based on our current findings, academics record their research output for the purpose of their own accounting to faculty, and not to increase the visibility of their research through open access.

Acknowledgments. We thank the UTS Library for their support of this paper.

\section{References}

1. Jantz, R.C., Wilson, M.C.: Institutional Repositories: Faculty Deposits, Marketing, and the Reform of Scholarly Communication. J. Acad. Librariansh. 34, 186-195 (2008)

2. Betz, S., Hall, R.: Self-Archiving with Ease in an Institutional Repository: Microinteractions and the User Experience. Inf. Technol. Libr. 34, 43-58 (2015)

3. Kim, J.: Motivations of Faculty Self-archiving in Institutional Repositories. J. Acad. Librariansh. 37, 246-254 (2011)

4. Bell, S., Foster, N.F., Gibbons, S.: Reference librarians and the success of institutional repositories. Ref. Serv. Rev. 33, 283-290 (2005) 
5. MacMillan, D.: Data sharing and discovery: What librarians need to know. J. Acad. Librariansh. 40, 541-549 (2014)

6. Armstrong, M.: Institutional repository management models that support faculty research dissemination. OCLC Syst. Serv. 30, 43-51 (2014)

7. Körber, N., Suleman, H.: Usability of Digital Repository Software: A Study of DSpace Installation and Configuration. In: Buchanan, G., Masoodian, M., and Cunningham, S.J. (eds.) Digital Libraries: Universal and Ubiquitous Access to Information: 11th International Conference on Asian Digital Libraries, ICADL 2008, Bali, Indonesia, December 2-5, 2008. Proceedings. pp. 31-40. Springer, Heidelberg (2008)

8. Van House, N.A., Butler, M.H., Ogle, V., Schiff, L.: User-centered iterative design for digital libraries. D-Lib Magazine. 2, (1996)

9. McKay, D.: Institutional repositories and their 'other' users: usability beyond authors. Ariadne. 52, (2007)

10. Aljohani, M., Blustein, J.: Personas Help Understand Users' Needs, Goals and Desires in an Online Institutional Repository. Int. J. Comput. Electr. Autom. Control Inf. Eng. 9, 629-636 (2015)

11. Koshiyama, D., de Pinho, A.L.S., Santa Rosa, J.G.: Analysis of Usability and Information Architecture of the UFRN Institutional Repository. In: Marcus, A. (ed.) Design, User Experience, and Usability: Interactive Experience Design: 4th International Conference, DUXU 2015, Held as Part of HCI International 2015, Los Angeles, CA, USA, August 2-7, 2015, Proceedings, Part III. pp. 197-207. Springer International Publishing, Cham (2015)

12. Law, E.L.-C., Roto, V., Hassenzahl, M., Vermeeren, A.P.O.S., Kort, J.: Understanding, scoping and defining user experience. Proc. 27th Int. Conf. Hum. factors Comput. Syst. - CHI 09. 719 (2009)

13. Blandford, A., Buchanan, G.: Usability of digital libraries: a source of creative tensions with technical developments. IEEE-CS Tech. Comm. Digit. Libr. 9 (2003)

14. Open Access Policy. http://www.gsu.uts.edu.au/policies/open-access.html

15. Stasko, J., Catrambone, R., Guzdial, M., McDonald, K.: An evaluation of spacefilling information visualizations for depicting hierarchical structures. Int. J. Hum. Comput. Stud. 53, 663-694 (2000)

16. Australian and New Zealand Standard Research Classification (ANZSRC), 2008. http://www.abs.gov.au/ausstats/abs@.nsf/0/ 6BB427AB9696C225CA2574180004463E

17. Thong, J., Hong, W., Tam, K.-Y.: Understanding user acceptance of digital libraries: what are the roles of interface characteristics, organisational context, and individual differences. Int. J. Hum. Comput. Stud. 57, 215-242 (2002)

18. Kupersmith, J.: Library Terms That Users Understand. UC Berkeley Libr. 1-36 (2012)

19. Nielsen, J., Molich, R.: Heuristic Evaluation of User Interfaces. In: Proceedings of the SIGCHI Conference on Human Factors in Computing Systems. pp. 249-256. ACM, New York, NY, USA (1990)

20. Shneiderman, B.: The Eyes Have It: A Task by Data Type Taxonomy for Information Visualizations. In: Proceedings of the 1996 IEEE Symposium on Visual Languages. p. 336-343. IEEE Computer Society, Washington, DC, USA (1996).

21. Nielsen, J., Landauer, T.K.: A Mathematical Model of the Finding of Usability Problems. In: Proceedings of the INTERACT '93 and CHI '93 Conference on Human Factors in Computing Systems. pp. 206-213. ACM, New York, NY, USA (1993) 